\title{
The third Bond
}

\section{When James Watson and Francis Crickunveiled their structure of DNA, one of the two kinds of base pair in the molecule was given two hydrogen bonds instead of three. Who spotted the third bond and when?}

\section{Simon Wain-Hobson}

It is a truth universally acknowledged that a guanine-cytosine (GC) base pair has three hydrogen bonds whereas adenine-thymine (AT) has two. But James Watson and Francis Crick didn't see it that wayback in 1953 when they published the structure of DNA. In their second DNA paper published in May of that year, the GC base pair is shown with only two hydrogen bonds (see top figure). So who spotted the third bond? When? And why was it initially passed over?

In his book The Double Helix, Watson notes that ${ }^{\alpha}$ The formation of a third hydrogen bond between guanine and cytosine was considered but rejected because a crystallographic study of guanine hinted that it would be very weak ${ }^{x}$.

The third hydrogen bond in a GC pair makes its first published appearance in a paper by Linus Pauling and Robert Corey in 1956 (see bottom figure). However, the first hint of the third bond in the scientific literature actually comes in a footnote to a paper published earlier that year by Jerry Donohue, a physical chemist and crystallographer.

In this paper ${ }^{2}$, which describes the possible ways in which pyridines and purines might hydrogen bond to one another, Donohue notes, "It has been pointed out by Professor Pauling that it is possible with only small distortion for guanine and cytosine to pair by formation of three hydrogen bonds... The formation of this additional hydrogen bond may confer extra stability on the Watson-CrickStructure." The respectful tone is understandable given that Pauling recommended Donohue's paper to the Proceedings of the National Academy of Sciences on 23 November, 1955. So Pauling had the third bond by the end of that year.

But what was the guanine crystal structure alluded to in The Double Helix that led Watson and Crick to reject the third bond? Their colleagues at the Cavendish Laboratory in Cambridge, under the direction of Lawrence Bragg, had been working on the structure of pyrimidines, purines and nucleosides since 1948, including adenine, guanine hydrochloride and a uracil derivative. Meanwhile, down in Birkbeck College, London, another group had published the structure of cytidine. So by spring 1953 initial structures of the four bases were either known or could be reasonably

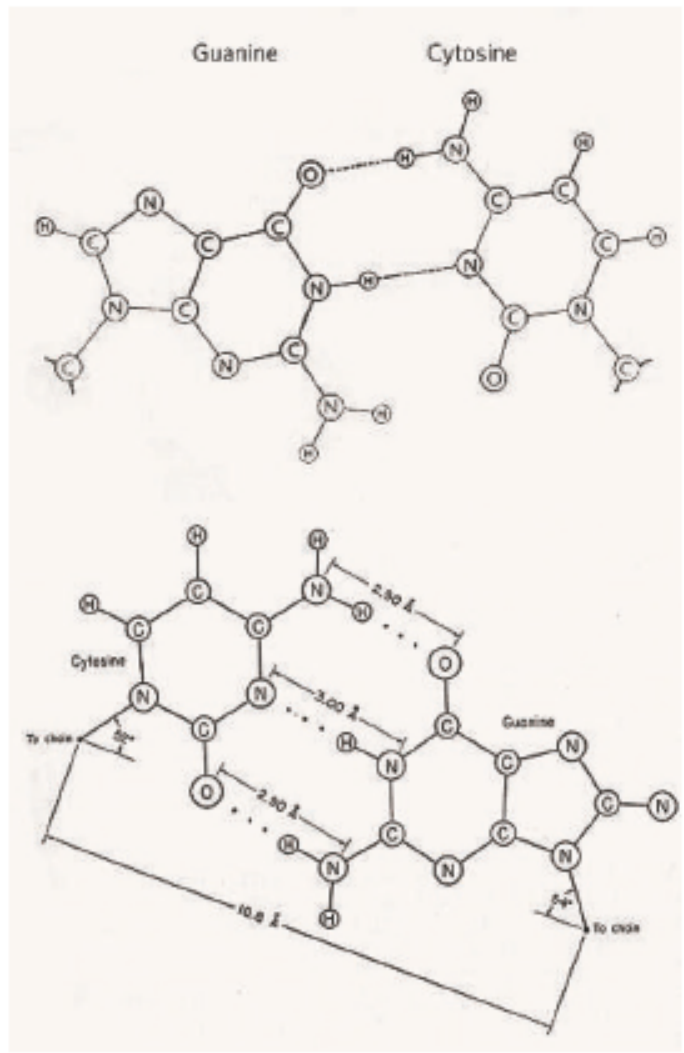

The third hydrogen bond in a guanine-cy tosine base pair (bottom) was missed in the 1953 description of DNA (top).

inferred. These data would have been available to Watson and Crick.

The adenine and guanine structures used in Watson and Crick's figure seem to be those determined by Bill Cochran and June Broomhead of the Cavendish Laboratory. Telltale signs are in the guanine structure - the bonds surrounding the keto and amino groups are irregular, distorting this part of the structure.

In Watson and Crick's figure, the hydrogen-donating amino group in the guanine base leans away from the keto acceptor group of cytidine (see top figure). Hydrogen bonds are at their strongest when the hydrogen atom and the donor and acceptor atoms are aligned linearly. Any third bond drawn on this figure would be at best weak with a 'kink' of about $18^{\circ}$ from this linear position, and would have been a little on the long side at 3.3 angstroms.

But why did Watson and Crick reject even a weak third bond? The answer may lie back in Donohue's 1956 paper $^{2}$. In that paper on hydrogen-bonding patterns between purines and pyrimidines, "a maximum deviation of $\mathrm{N}-\mathrm{H}$...X $\mathrm{X}$ from linearity of about $15^{\circ}$ was allowed".

Donohue shared the same office as Watson and Crick at the Cavendish Laboratory. It was he who advised Watson over which tautomeric forms of pyrimidines and purines to use in their DNA model.

The acknowledgement, ${ }^{\alpha} \mathrm{We}$ are much indebted to Dr. Jerry Donohue for constant advice and criticism, especially in inter-atomic distances," appears at the end of the first DNA paper - indeed before mention of Maurice Wilkins and Rosalind Franklin, both key players in the discovery of DNA's structure. So it may be presumed that Watson and Crick deferred to Donohue and cut the third bond.

Pauling and Corey, however, arrived at the right structure thanks to a strong dose of structural common sense. They note that the structure for guanine contains "a small error $^{n}$ in that angles of the bonds adjacent to the keto group are irregular.

While working from the literature, they made many "reasonable arguments based upon considerations of electronic structure , one of which was that equal bond angles surround the keto and amino groups.

Using a "reasonable" structure for guanine, the third bond falls into place like a charm. Indeed, the third bond proved to be every bit as good as any of the other hydrogen bonds in AT and GC pairs coming in at 2.9 angstroms, the $\mathrm{N}-\mathrm{H}$... $\mathrm{O}$ hydrogen bond being essentially linear. The bottom line is that there is a trace of Pauling in the double helix.

1953 was an excellent year - the structure of DNA, the Miller-Urey experiment, and the death of Stalin. And of course with Casino Royale the other Bond, James Bond, first stepped off the page in 1953.

Simon Wain-Hobson is at the Pasteur Institute, Paris.

FURTHER READING

1. Pauling L\& Corey, R. B. Arch. Biochem. Biophys.65, 164-181 (1956).

2. Donohue, LProc. Natl. Acad Sci. USA 42, 60-65 (9956). 